\title{
THE GENERAL FORM OF HYPERGEOMETRIC SERIES OF TWO VARIABLES ${ }^{1}$
}

\author{
A. ERDÉLYI
}

1. A formal hypergeometric series of two variables is defined ${ }^{2}$ as a formal double power series

$$
\sum a_{m n} x^{m} y^{n}
$$

for whose coefficients the ratios

$$
a_{m+1, n} / a_{m, n}=f(m, n) \quad \text { and } \quad a_{m, n+1} / a_{m, n}=g(m, n)
$$

are fixed rational functions of $m$ and $n$. The most general form of such a series has been a subject of controversy and as far as I know the explicit result is given here for the first time.

Appell, Birkeland, Horn, Kampé de Fériet, Mellin, and others have considered series for which the coefficients are gamma products, that is to say they are of the form

$$
\gamma_{m n}=\prod_{i} \Gamma\left(\alpha_{i}+u_{i} m+v_{i} n\right) / \Gamma\left(\alpha_{i}\right)
$$

where the $\alpha_{i}$ are arbitrary (real or complex) constants, and the $u_{i}$ and $v_{i}$ are arbitrary integers which may be positive, zero, or negative. The question arises whether the series considered by Horn and other writers are the most general hypergeometric series of two variables. Clearly, $f$ and $g$ must satisfy the relation

$$
f(m, n) g(m+1, n)=f(m, n+1) g(m, n)
$$

for all $m, n=0,1,2, \cdots$, because each of the two sides is equal to $a_{m+1, n+1} / a_{m n}$; and conversely, it is easy to show that any pair $f, g$ of rational functions satisfying (3) generates a series (1) for which

$$
a_{m n}=a_{00} \prod_{i=0}^{m-1} f(i, 0) \prod_{j=0}^{n-1} g(m, j)
$$

Everything, then, turns on the rational solutions of the functional equation (3).

Birkeland stated ${ }^{3}$ that every rational solution of (3) can be de-

Presented to the International Congress of Mathematicians, September 1, 1950; received by the editors May 25, 1950.

1 Research sponsored by the Office of Naval Research.

2 J. Horn, Math. Ann. vol. 34 (1889) pp. 544-600.

${ }^{3}$ R. Birkeland, C. R. Acad. Sci. Paris vol. 185 (1927) pp. 923-925. 
composed into linear factors, and this would seem to establish essentially (2) as the most general form of coefficients of hypergeometric series of two variables. Now, clearly, Birkeland's statement disregards certain obvious exceptions to his rule. For instance, if $a_{m n}$ itself is a fixed rational function of $m$ and $n$, it may be impossible to factorize $f$ and $g$ into linear factors: $a_{m n}=m^{2}+n$ illustrates this case. ${ }^{4}$

Accordingly, $\mathrm{Ore}^{5}$ noted that Birkeland's theorem is not true in general, and ${ }^{6}$ gave a detailed analysis of the rational solutions of the functional equation (3). Ore remarks ${ }^{6}$ (p. 325) that his results should enable one to construct the most general form of a hypergeometric series of two variables, but as far as I know this construction has not been carried out.

In this note I shall use Ore's results to show that apart from a factor $a^{m} b^{n}$, the most general form of $a_{m n}$ is the product of a fixed rational function of $m$ and $n$ with a gamma product. Moreover, the most general hypergeometric series of two variables results from the operation of a rational differential operator on a series of the Horn-Birkeland type so that the latter series appear to be essentially the parent series for all hypergeometric series of two variables.

2. With Ore we shall use the symbol $\prod_{j=0}^{k-1} h(j)$ for any integer $k$ to mean

$$
\begin{array}{cl}
h(0) h(1) \cdots h(k-1) & \text { if } \quad k>0 \\
1 & \text { if } \quad k=0 \\
\{h(-1) h(-2) \cdots h(k)\}^{-1}, & \text { if } \quad k<0
\end{array}
$$

Also, let $A_{1}, \cdots, A_{t}, B_{1}, \cdots, B_{t}$ be (positive or negative) nonzero integers, let $C$ be any (real or complex) number, and put $a_{1}=b_{1}=0$, $a_{i}=A_{1}+\cdots+A_{i-1}, \quad b_{i}=B_{2}+\cdots+B_{i}, \quad i=2,3, \cdots, t, \quad A=A_{1}$ $+\cdots+A_{t}, B=B_{1}+\cdots+B_{t}$.

Ore's results can be summarized as follows:

(i) If $A \neq 0, B \neq 0$, and $L(m, n)=A m+B n+C$, then

$$
\begin{aligned}
& f(m, n)=\prod_{i=1}^{t} \prod_{j=0}^{A_{i}-1} L\left(m-b_{i}, n+a_{i}+j\right), \\
& g(m, n)=\prod_{i=1}^{t} \prod_{j=0}^{B_{i}-1} L\left(m-b_{i}+j, n+a_{i}\right)
\end{aligned}
$$

It is quite easy to see at which stage the exceptions slip through the net of Birkeland's analysis. As a preliminary step, he divides (3) by the common factors of $f(m, n)$ and $g(m, n)$ and also by the common factors of $f(m, n+1)$ and $g(m+1, n)$. These common factors are precisely the ones which need not factorize into linear factors.

b O. Ore, C. R. Acad. Sci. Paris vol. 189 (1929) pp. 1238-1240.

6 O. Ore, J. Math. Pures Appl. (9) vol. 9 (1930) pp. 311-326. 
is a solution of the functional equation (3).

(ii) If $A=B=0$ and $H(m, n)$ is any polynomial in $m$ and $n$, then

$$
\begin{aligned}
& f(m, n)=\prod_{i=1}^{t} \prod_{j=0}^{A_{i}-1} H\left(m-b_{i}, n+a_{i}+j\right), \\
& g(m, n)=\prod_{i=1}^{t} \prod_{j=0}^{B_{i}-1} H\left(m-b_{i}+j, n+a_{i}\right)
\end{aligned}
$$

is a solution of (3).

(iii) Apart from factors which depend on $m$ only or on $n$ only, every rational solution of the functional equation (3) is a product of a finite number of factors of the form (4) or (5).

We shall now attempt to analyze the implications of these results; in particular, we shall try to find the corresponding forms of the coefficients $a_{m n}$.

A factor of $f$ which depends on $m$ only is necessarily of the form $K \prod_{i}\left(\alpha_{i}+m\right) / \prod_{j}\left(\beta_{j}+m\right)$ where $K, \alpha_{i}, \beta_{j}$ are constants. The corresponding factor of $a_{m n}$ is, apart from a constant,

$$
K^{m} \prod_{i} \Gamma\left(\alpha_{i}+m\right) / \prod_{i} \Gamma\left(\beta_{j}+m\right),
$$

and hence essentially a gamma product. A factor of $f$ which is independent of $m$ is necessarily also independent of $n$ and hence a constant. Similar statements hold for $g$. Thus, in the solution of the functional equation (3), factors which depend on $m$ only or on $n$ only correspond essentially to gamma products in $a_{m n}$.

3. Let us analyze (5). In order to do so, take in turn $i=1,2, \cdots, t$; and for each $i$ the numbers $n+a_{i}+j$ where $j=0,1, \cdots, A_{i}-1$ if $A_{i}>0$, or $j=-1,-2, \cdots, A_{i}$ if $A_{i}<0$. In this manner we obtain a sequence of $\sum\left|A_{i}\right|$ integers, each of which differs from the preceding one by unity, except at the transition from a positive $A_{i}$ to a negative $A_{i+1}$ or from a negative $A_{i}$ to a positive $A_{i+1}$ when an integer is repeated. Also, the last integer, which is $n+a_{t}+A_{t}-1$ if $A_{t}>0$, or $n+a_{t}+A_{t}$ if $A_{t}<0$, differs by unity from the first one, which is $n$ if $A_{1}>0$, or $n-1$ if $A_{1}<0$ except when $A_{1}$ and $A_{t}$ have opposite signs, when the first and last integer of the sequence are equal. Hence each integer which appears in the sequence at all appears an even number of times of which one half is associated with a positive $A_{i}$ and the other half with a negative $A_{i}$. Suppose that $\beta+n$ is one of the integers of the sequence, that it belongs to a positive $A_{i}$ when $-b_{i}=\alpha$ and to a negative $A_{i}$ when $-b_{i}=\alpha^{\prime}$. In $f(m, n)$ we shall then have the factor

$$
H(m+\alpha, n+\beta) / H\left(m+\alpha^{\prime}, n+\beta\right)
$$


which will correspond to the factor

$$
\prod_{j=0}^{\alpha-\alpha^{\prime-1}} H\left(m+\alpha^{\prime}+j, n+\beta\right)
$$

in $a_{m n}$. (This product like all similar ones is to be interpreted in accordance with the convention set out in $\$ 2$.) Since (5) satisfies (3), the corresponding factor,

$$
\prod_{j=0}^{\alpha-\alpha^{\prime-1}} H\left(m+\alpha^{\prime}+j, n+\beta+1\right) / \prod_{j=0}^{\alpha-\alpha^{\prime}-1} H\left(m+\alpha^{\prime}+j, n+\beta\right),
$$

is bound to be present in $g$.

Dealing in a similar manner with all the factors of $f(m, n)$ and $g(m, n)$, it is seen that all contributions to $a_{m n}$ which arise out of expressions of the form (5) are factors of the form $\{H(m+\alpha, n+\beta)\}^{\gamma}$ where $\alpha, \beta, \gamma$ are integers; in other words that (5) contributes to $a_{m n}$ a factor which is a (fixed) rational function of $m$ and $n$.

4. We now turn to (4). As in the previous section, consider the sequence of integers $n+a_{i}+j$ where $i=1,2, \cdots, t$ and, for each $i$, $j=0,1, \cdots, A_{i}-1$ if $A_{i}>0$, or $j-=1,-2, \cdots, A_{i}$ if $A_{i}<0$. As in the previous section, each integer in this sequence differs from its predecessor by unity, except at the transition from a positive $A_{i}$ to a negative $A_{i+1}$ or from a negative $A_{i}$ to a positive $A_{i+1}$; but now the last integer of the sequence, which is $n+A-1$ if $A_{t}>0$, or $n+A$ if $A_{t}<0$, differs from the first one which is $n$ if $A_{1}>0$, or $n-1$ if $A_{1}<0$, by $A \pm 1$ if $A_{1}$ and $A_{t}$ are of the same sign, or by $A$ if $A_{1}$ and $A_{t}$ are of opposite signs. There may be (and if among the $A_{i}$ there are both positive and negative numbers there will be) integers which occur more than once in the sequence, very much in the same way as in $\$ 3$, and give rise to a factor of $a_{m n}$ which is a fixed rational function of $m$ and $n$; but after these integers have been removed from the sequence of $\sum\left|A_{i}\right|$ integers, there will remain the $|A|=\left|\sum A_{i}\right|$ integers $n, n+1, \cdots, n+A-1$ if $A>0$ or $n-1, n-2, \cdots, n+A$ if $A<0$.

If $A$ and $B$ are coprime, the sequence of least non-negative residues to modulus $A$ of the numbers $j B$, where $j=0,1, \cdots, A-1$ if $A>0$ or $j=-1,-2, \cdots, A$ if $A<0$, is a permutation of the sequence of $j$, and the $|A|$ factors of $f(m, n)$ may be written as

$$
\prod_{j=0}^{A-1}\left\{A\left(m+\alpha_{i}\right)+B n+C+j\right\}
$$


or

$$
\begin{aligned}
& \prod_{j=0}^{A-1} \frac{A\left(m+\alpha_{j}\right)+B n+C+j}{A m+B n+C+j} \prod_{j=0}^{A-1}(A m+B n+C+j) \\
&=f_{1}(m, n) f_{2}(m, n)
\end{aligned}
$$

with some integers $\alpha_{j}$. The first factor, $f_{1}(m, n)$, is similar to the one already encountered in $\$ 4$ and gives rise to a fixed rational factor in $a_{m n}$, and the last factor, $f_{2}(m, n)$, indicates the presence in $a_{m n}$ of $\Gamma(A m+B n+C)$.

If $A$ and $B$ have a common divisor, the argument is similar. Let $d$ be the highest common divisor, $A=a d$ and $B=b d$. The sequence of numbers $j b, j=0,1, \cdots, A-1$ if $A>0$, or $j=-1,-2, \cdots, A$ if $A<0$ can now be decomposed into $d$ subsequences of $|a|$ numbers each so that the least non-negative residues to modulus $a$ of each subsequence form a rearrangement of the sequence $0,1, \cdots, a-1$ if $a>0$, or of $-1,-2, \cdots, a$ if $a<0$. In this case a similar argument to the one used above shows that the corresponding factor of $a_{m n}$ is the product of $d^{A m+B n}$, of a fixed rational function of $m$ and $n$, and of $\{\Gamma(a m+b n+c / d)\}^{d}$.

Thus, (4) contributes only factors to $a_{m n}$ which are of the form stated in $\$ 1$, and we see that the most general form of the coefficients of a hypergeometric series of two variables is

$$
a_{m n}=h^{m} k^{n} R(m, n) \gamma_{m n}
$$

where $h, k$ are constants, $R$ is a rational function of $m$ and $n$, and $\gamma_{m n}$ is a gamma product.

5. Let $\delta \equiv x \partial / \partial x$ and $\delta^{\prime} \equiv y \partial / \partial y$ be the operators of differentiation with respect to $\log x$ and $\log y$. Now,

$$
\delta\left(x^{m} y^{n}\right)=m x^{m} y^{n}, \quad \delta^{\prime}\left(x^{m} y^{n}\right)=n x^{m} y^{n}
$$

and hence

$$
\sum R(m, n) \gamma_{m n}(h x)^{m}(k y)^{n}=R\left(\delta, \delta^{\prime}\right) \sum \gamma_{m n}(h x)^{m}(k y)^{n},
$$

showing that any (formal) hypergeometric series of two variables is the result of operating (formally) on a hypergeometric series of the Horn-Birkeland type with a rational differential operator with constant coefficients. This result is important in that it enables one to reduce the study of all hypergeometric series of two variables essentially to the series of the Horn-Birkeland type. A further reduction, to a yet smaller number of series, is possible and has been carried 
out for series of order two. ${ }^{7}$

If a hypergeometric series is convergent for certain nonvanishing $x$ and $y$, it defines a hypergeometric function. Our last result suggests that the presence of the factor $R(m, n)$ is without influence on the fact, or domain, of convergence. This can be seen directly as follows: $r, s$ are said to be associated radii of convergence of (1) if

$$
\lim \sup \left\{\left|a_{m n}\right| r^{m} s^{n}\right\}^{1 /(m+n)}=1 \text { as } m, n \rightarrow \infty .
$$

Now, for every rational function $R$,

$$
\lim \sup |R(m, n)|^{1 /(m+n)}=1,
$$

and hence (7) remains true if a factor of the form $R(m, n)$ is omitted from, or added to $a_{m n}$.

$\mathrm{Ore}^{6}$ (p. 311) states that his results can be generalized to hypergeometric functions of more than two variables. A similar possibility exists for the conclusions drawn in this note from his results.

California Institute of Technology

${ }^{7}$ A. Erdélyi, Proc. Roy. Soc. Edinburgh. Ser. A vol. 62 (1948) pp. 378-385, and Acta Math. vol. 83 (1950) pp. 131-164. 